\title{
Avances en el diseño y cálculo de columnas de grava. Parte I: Ejemplo de cálculo
}

\section{Recent advances in the design and calculation methods of stone columns. Part I: A calculation example}

$\underline{\text { Jorge Castro }}^{(*)}$

\section{RESUMEN}

Este artículo recoge algunos aspectos teóricos recientes relativos a columnas de grava que pueden resultar útiles para su diseño y cálculo. En esta primera parte, se presenta un ejemplo de cálculo de reducción de asientos y aceleración de tiempos de consolidación utilizando diferentes métodos. De forma general, los métodos de cálculo utilizados han proporcionado resultados adecuados si se tienen en cuenta las limitaciones y posibilidades de cada una de ellos. Los métodos semi-empíricos son sencillos porque proporcionan la reducción de asiento en función principalmente del factor de sustitución. Los métodos analíticos desarrollados recientemente basados en la celda unidad permiten considerar la influencia de otros parámetros, pero normalmente la información disponible respecto a su valor es limitada. Finalmente, los cálculos por elementos finitos proporcionan una gran flexibilidad en el análisis, pero es necesaria una adecuada comprensión de las hipótesis, modelos constitutivos y parámetros empleados.

Palabras clave: columnas de grava; asiento; consolidación; cálculo; diseño; elementos finitos; suelo blando.

\section{ABSTRACT}

This paper comprises recent theoretical advances that may be useful for the design and analysis of stone columns. In this first part, a calculation example is developed using different methods to study the settlement reduction and the consolidation process. The calculation methods used for this example have generally provided satisfactory results, considering the possibilities and limitations of each method. Semi-empirical methods are easy to use because the settlement reduction is obtained directly, or almost directly, from the area replacement ratio. Recently developed analytical methods based on the unit cell model consider the influence of other parameters, but information about the value of those input parameters is usually limited. Finally, finite element analyses are highly flexible, but a deep understanding of the calculation hypotheses, constitutive models and input parameters is required.

Keywords: stone columns; settlement; consolidation; calculation; design; finite elements; soft soil.

(*) Dr. Ing. Caminos, C y P. Profesor Titular. Universidad de Cantabria, Santander (España).

Persona de contacto/Corresponding author: castrogj@unican.es (J. Castro)

ORCID: https://orcid.org/oooo-0001-5815-9995 (J. Castro)

Cómo citar este artículo/Citation: Castro, Jorge (2020). Avances en el diseño y cálculo de columnas de grava. Parte I: Ejemplo de cálculo. Informes de la Construcción, 72(560): e362. https://doi.org/10.3989/ic.71911

Copyright: (C) 2020 CSIC. Este es un artículo de acceso abierto distribuido bajo los términos de la licencia de uso y distribución Creative Commons Reconocimiento 4.0 Internacional (CC BY 4.0). 


\section{INTRODUCCIÓN}

Esta publicación deriva de la ponencia realizada por el autor en las $19^{\text {as }}$ Jornadas Técnicas SEMSIG-AETESS, celebradas en Madrid el día 21 de febrero de 2019. Este artículo se centra en las columnas de grava, y más concretamente en el cálculo de asientos y reducción del tiempo de consolidación. Dentro de esta temática, se consideran aspectos teóricos, pero con un enfoque práctico para que puedan resultar útiles en el diseño y cálculo de columnas de grava. Debido a su extensión, se ha divido en dos partes.

En esta primera parte se recoge un ejemplo de cálculo en el que se comparan diferentes metodologías. En la segunda parte (1), se presentan comentarios sobre grupos de columnas y la problemática que deriva de los asientos por consolidación secundaria. Así, se presenta a continuación, una breve introducción a la técnica (Apartado 2), un ejercicio de predicción que se toma como punto de partida (Apartado 3), los datos del ejercicio de cálculo (Apartado 4) y el cálculo de asientos sin y con columnas (Apartados 6 y 7, respectivamente). Finalmente, se presenta una discusión y comparación de los resultados obtenidos con las diferentes metodologías de cálculo (Apartado 8) y se resumen las principales conclusiones.

\section{COLUMNAS DE GRAVA: ASPECTOS BÁSICOS}

Las columnas de grava surgieron como una extensión de las técnicas de vibrocompactación profunda en suelos finos y constituyen uno de los métodos habituales de mejora del terreno para la cimentación de terraplenes o estructuras en suelos blandos. Son perforaciones verticales en el terreno, que se rellenan en sentido ascendente con grava introducida mediante un vibrador, que va realizando su compactación (p. ej., (2)). La inclusión de grava en un suelo fino permite mejorar: la capacidad portante, la estabilidad al deslizamiento, el asiento final, el tiempo de consolidación y el potencial de licuefacción. En suelos muy blandos que no proporcionan un adecuado confinamiento lateral, una solución bastante extendida consiste en rodear las columnas con un geosintético (p. ej., (3)).

La ejecución de las columnas altera el suelo circundante, generándose incrementos de presión intersticial y de tensiones horizontales. Si los incrementos de presión intersticial se disipan antes de la ejecución del elemento a cimentar, éstos suelen tener un efecto positivo y por ello se suelen despreciar en el diseño. No obstante, cuando estos excesos de presión intersticial no se hayan disipado o cuando se trate de un suelo susceptible al remoldeo generado por la instalación, es necesario tener en cuenta la disminución de la resistencia al corte sin drenaje y comprobar la correcta ejecución de la columna (p. ej., (4-8)).

Bajo cargas de gran extensión (p. ej., terraplenes), la mejora tiene un carácter masivo, distribuyéndose las columnas en una malla uniforme. La existencia de un gran número de columnas, en una situación análoga de carga y geometría (p. ej., en la parte central de un terraplén), permite utilizar un modelo geométrico simplificado que se denomina de «celda unidad» y consiste en una única columna con el área tributaria o suelo circundante correspondiente. Debido a las condiciones de simetría, en el contorno exterior de la celda unidad se permiten desplazamientos y flujo de agua únicamente en la dirección vertical. Para poder estudiar el problema de forma bidimensional, es decir, en simetría axial, es habitual transformar el área tributaria (de sección hexagonal o cuadrada para mallas de columnas triangulares y cuadradas, respectivamente) en una sección circular de igual área. Así, el diámetro exterior de la celda unidad es $D_{e}=1.05^{-1.13 s}$ para mallas triangulares y cuadradas, respectivamente, donde s es la separación entre ejes de columnas (Figura 1).

El parámetro más determinante en el análisis de tratamientos con columnas de grava es el factor de sustitución $\left(a_{r}\right)$ que representa el porcentaje de terreno blando sustituido o desplazado por las columnas:

$$
a_{r}=A_{C} / A_{T}
$$

donde $A_{C}$ es el área ocupada por las columnas y $A_{T}$ el área cargada. La notación aquí empleada es habitual en muchas publicaciones científicas, pero difiere de la utilizada por la Guía de Cimentaciones en Obras de Carretera (GCOC) (9) y las Recomendaciones Geotécnicas para Obras Marítimas y Portuarias (ROM 0.5-05) (10).

La reducción de asiento alcanzada con un tratamiento con columnas de grava se suele expresar mediante el factor de reducción de asientos:

$$
\beta=s_{z} / s_{z o}
$$
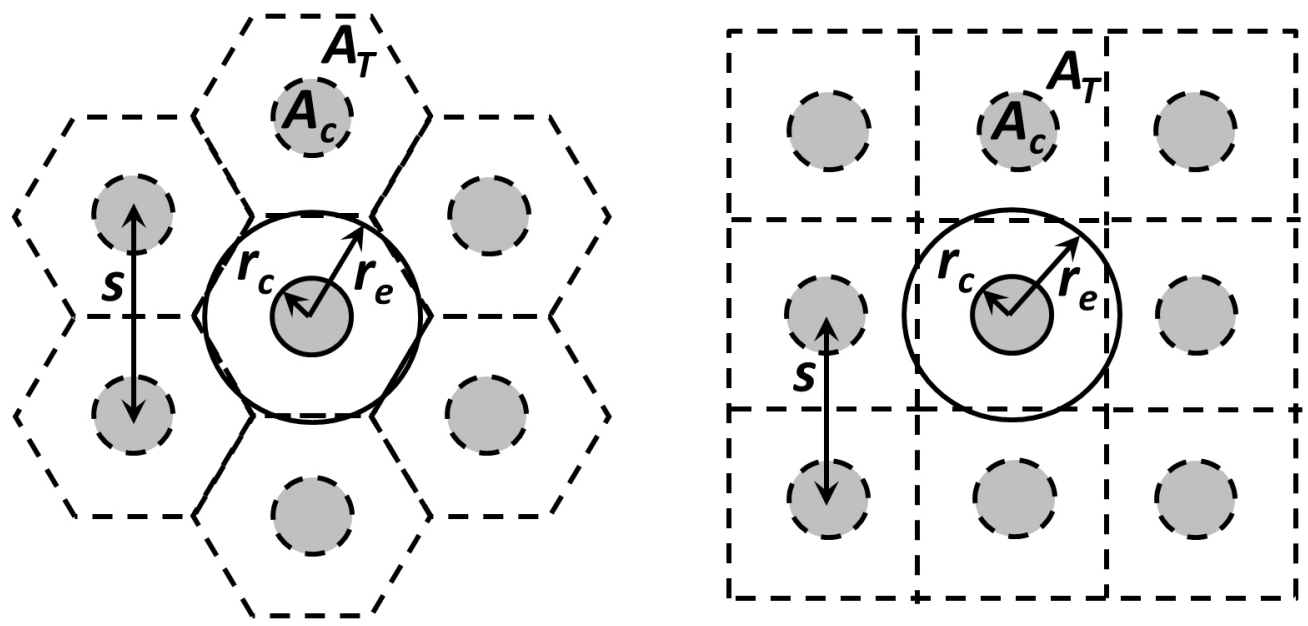

Figura 1. Simplificación de la celda unidad a simetría axial. 


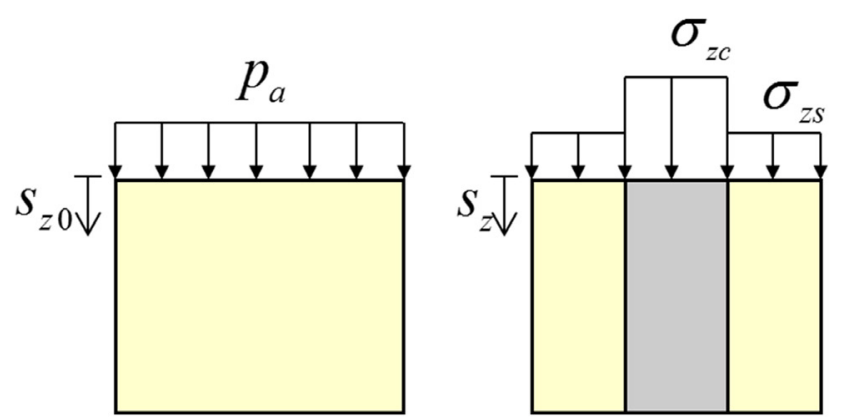

Figura 2. Esquema explicativo de los factores de reducción de asientos y de concentración de la carga.

donde $s_{z}$ y $s_{z o}$ son los asientos que se producen con y sin columnas, respectivamente (Figura 2). Algunos autores (p. ej., (11)) utilizan el inverso de la reducción de asientos, denominado factor de mejora $(n)$. Otro parámetro que también expresa la mejora proporcionada por las columnas es el factor de concentración de tensiones $(S C F)$, que recoge la mayor capacidad de las columnas para tomar la carga y descargar el suelo blando circundante. Así, se expresa como el cociente entre el incremento de tensión vertical en la columna $\left(\Delta \sigma_{z c}\right)$ y en el suelo $\left(\Delta \sigma_{z S}\right)$ :

$$
S C F=\Delta \sigma_{z c} / \Delta \sigma_{z s}
$$

\section{EJERCICIO DE PREDICCIÓN}

El objetivo de este artículo es analizar un caso de un terraplén apoyado en un suelo blando mejorado con columnas de grava mediante diferentes métodos de cálculo. Como punto de partida, se toma el ejercicio de predicción realizado durante el Simposio Internacional sobre Mejora del Terreno en Paris en el año 2004 (p. ej., (12-14)). El ejercicio se basaba en un terraplén experimental construido en el año 2003 en Francia, del que se disponía de medidas de asientos y diversos ensa- yos geotécnicos: edómetros, triaxiales, presiómetros y ensayos de molinete. No se disponía de otros ensayos de campo como, por ejemplo, ensayos de penetración estática (CPTU) o dilatómetro plano (DMT), que hubieran sido útiles. Aunque estrictamente no era una predicción «a priori» (Clase A, (15)) porque ya se disponía de las medidas de asientos, sólo los organizadores conocían estas medidas.

La geometría del terraplén se muestra en la Figura 3. Debido a que el objetivo no era analizar las diferencias derivadas de la interpretación de los resultados de la campaña geotécnica, los resultados de la misma se proporcionaron ya interpretados (13). El terreno de apoyo estaba formado por un relleno antiguo superficial de $1 \mathrm{~m}$ de espesor, bajo el que aparecía un aluvial, con dos partes diferenciadas: una limosa y otra arenosa. A $9 \mathrm{~m}$ de profundidad aparecía un sustrato duro que se puede considerar incompresible. Las columnas tenían una longitud de $L_{c}=6 \mathrm{~m}$, para empotrarse en el aluvial arenoso.

El diámetro medio de las columnas empleadas era de $D_{c}=0.9 \mathrm{~m}$ y se disponían en una malla triangular con un espaciamiento entre ejes de columnas de $2.15 \mathrm{~m}$. Para el ejercicio de predicción, se proporcionaron también las características de la grava (13).

El relleno del terraplén se realizó con grava, cuyas propiedades se recogen en (13). Para el ejercicio de predicción, se consideró un ritmo de elevación del terraplén simplificado, elevándose el terraplén de manera continua hasta $6 \mathrm{~m}$ en 40 días, y manteniendo posteriormente constante su altura hasta 160 días (12).

El perfil de asientos medido y predicho por los diferentes participantes al cabo de 160 días se muestra en la Figura 4. Los métodos de cálculo utilizados variaban desde métodos analíticos sencillos basados en la «celda unidad», es decir, unidimensionales (líneas horizontales en la Figura 4) hasta

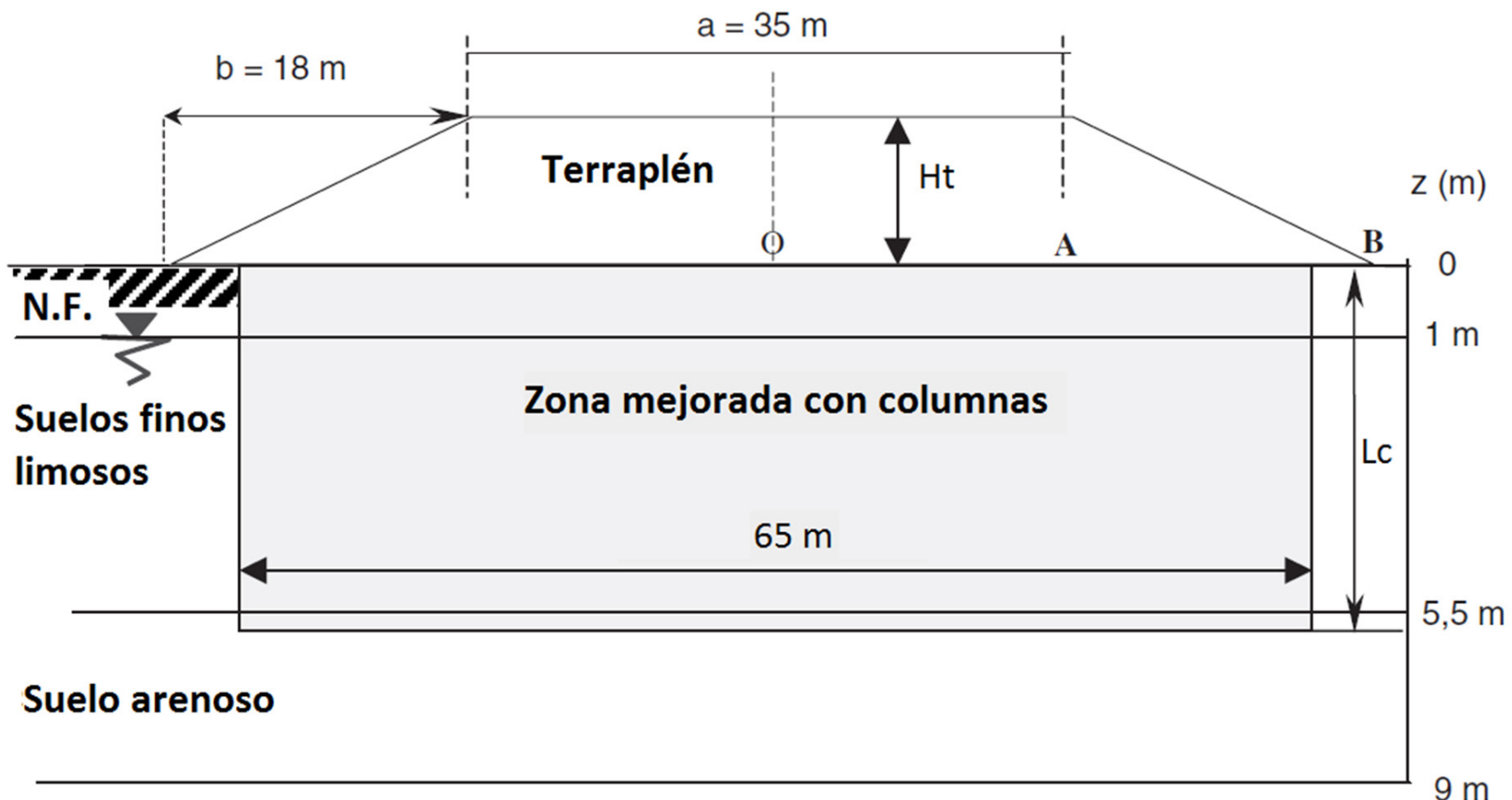

Sustrato incompresible

Figura 3. Sección transversal tipo del ejercicio de predicción (adaptado de (13)). 


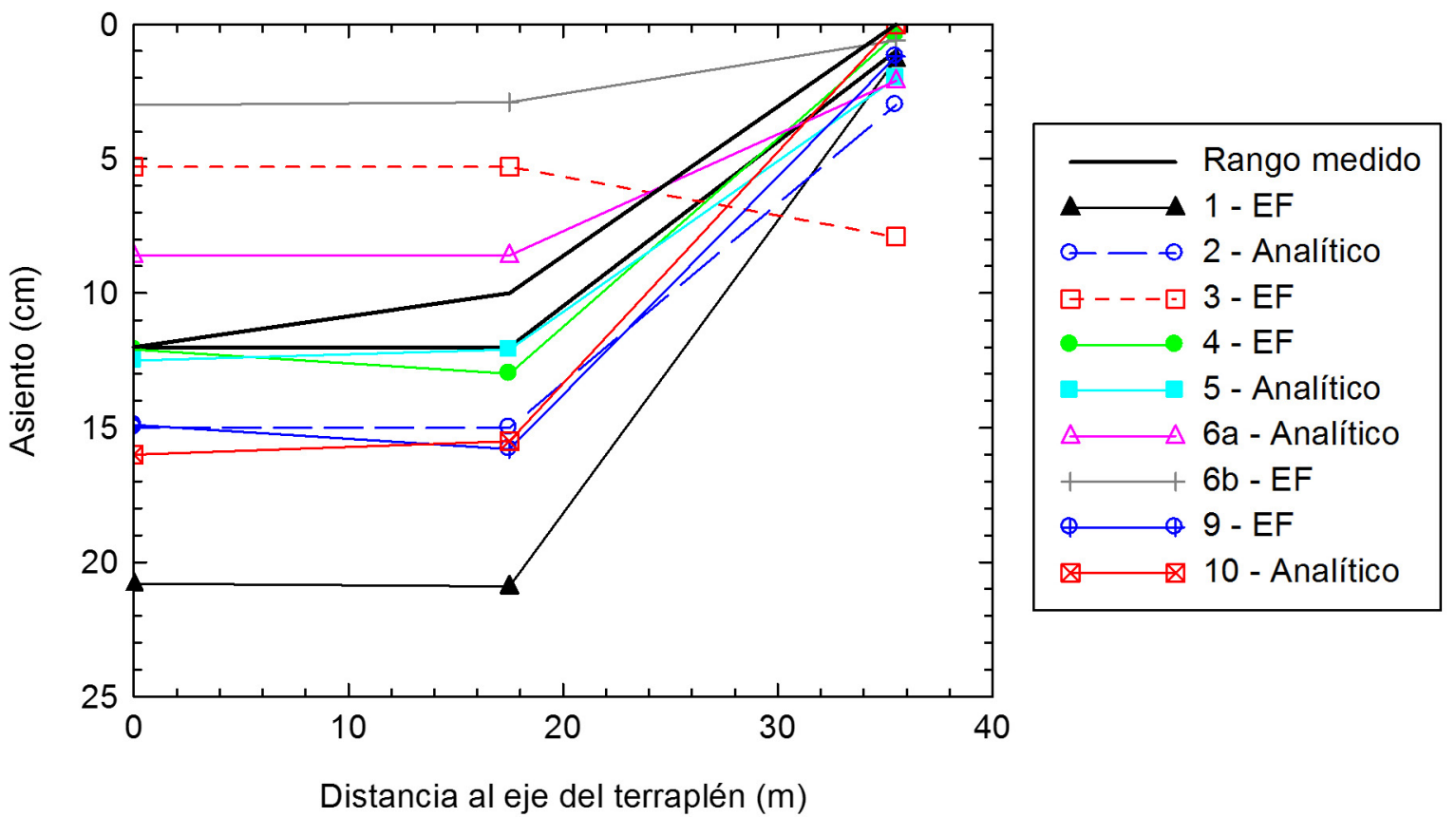

Figura 4. Asientos medidos y predichos al cabo de 160 días (adaptado de (12)).

métodos numéricos en deformación plana y tridimensionales (rebanada de columnas).

Las principales conclusiones que se pueden extraer de este ejercicio de predicción son las siguientes:

- Se observa una gran variabilidad en los resultados predichos (Figura 4). Sin embargo, una parte importante de esta variabilidad se puede atribuir a la variabilidad de los parámetros geotécnicos utilizados y no a los métodos de cálculo empleados. Por ejemplo, la variabilidad en la predicción del asiento sin columnas era también importante.

- El parámetro más importante en la predicción de asientos era la deformabilidad del estrato limoso. En base a los parámetros proporcionados, existían dos alternativas: utilizar el módulo de Menard $\left(E_{M}\right)$ o el coeficiente de compresión noval $\left(C_{C}\right)$ del modelo edométrico.

- El módulo de Menard $\left(E_{M}\right)$ es desde un punto de vista teórico equivalente al módulo de Young $(E)$. Sin embargo, la experiencia acumulada en Francia de predicción de asientos utilizando el ensayo presiométrico (p. ej., (16)) ha demostrado que es necesario aplicar un factor corrector $(\alpha)$ a $E_{M}$ para no sobreestimar los asientos $\left(E_{M} / E_{m}=\alpha\right)$, donde $E_{m}$ es el módulo edométrico. La normativa francesa (17) proporciona valores tabulados y aproximados de este factor corrector.

- Para el suelo aluvial limoso, los parámetros de deformabilidad y consolidación obtenidos del ensayo edométrico $\left(C_{C}\right.$ y $c_{v}$ ) son conservadores a la vista de los asientos medidos. Esto suele deberse al sesgo en el tallado de probetas para el ensayo edométrico. En estos casos (p. ej., depósitos aluviales), es recomendable realizar ensayos «in situ» como, por ejemplo, el ensayo de penetración estática CPTU, con ensayos de disipación a diferentes profundidades para obtener resultados más realistas del coeficiente de consolidación radial.

- Para un único tiempo intermedio (p. ej., 160 días, Figura 4), la predicción puede reproducir los resultados medidos a pesar de ser errónea, porque el efecto de una sobre- estimación de la deformabilidad $\left(C_{C}\right)$, se puede compensar con una consolidación más lenta (subestimación de $c_{v}$ ) (Figura 5).

- Los modelos de «celda unidad» (unidimensionales) son suficientes para predecir el asiento en el centro del terraplén. Para análisis más avanzados, es necesario ir a modelos numéricos tridimensionales de una rebanada de columnas (13).

\section{DATOS DE PARTIDA}

En base al ejemplo de predicción anterior, se plantean aquí los datos de partida del ejemplo de cálculo que se va a desarrollar. El objetivo es calcular:

- Valor del asiento final en el centro del terraplén.

- Asiento alcanzado al cabo de 50 días.

Para simplificar la secuencia de elevación del terraplén, se plantea la elevación instantánea del terraplén hasta una altura final de $6 \mathrm{~m}$. Los parámetros adoptados se resumen en la Figura 6. Para la deformabilidad de los suelos granulares se adoptan parámetros elásticos y para los limos, parámetros edométricos. El coeficiente de consolidación en los limos se asume anisótropo y, de forma conservadora, se toma su valor horizontal como el doble del vertical. La resistencia

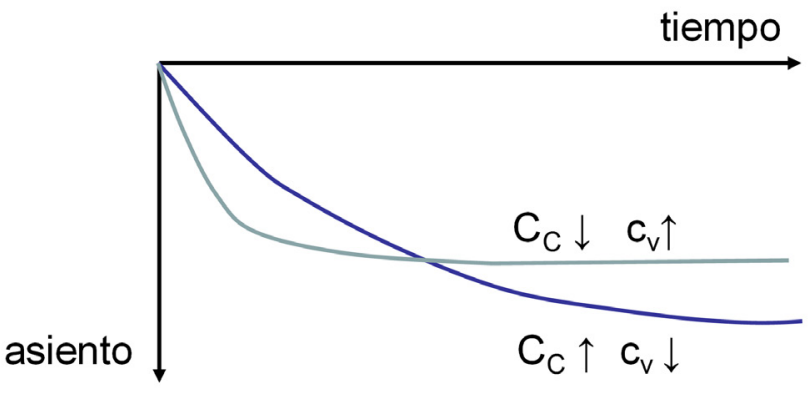

Figura 5. Evolución del asiento para dos pares de valores $C_{C} \mathrm{y} c_{v}$. 


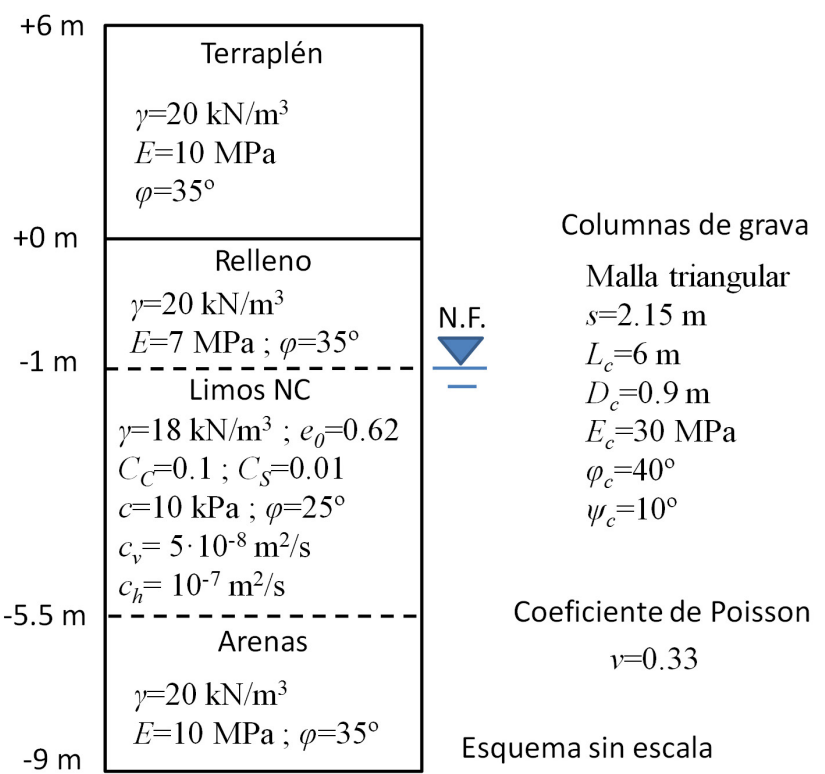

Figura 6. Datos de partida del ejercicio de cálculo.

de los suelos se fija mediante el criterio de rotura de MohrCoulomb. Para la grava de las columnas, se fijan parámetros resistentes y deformacionales para facilitar la comparación entre los diferentes métodos, a pesar de que estos parámetros no suelen conocerse. De la misma forma, para facilitar el análisis, se fija un coeficiente de Poisson general $(v=0.33)$ para todos los terrenos presentes.

Teniendo en cuenta que la malla de las columnas es triangular, el diámetro del área tributaria equivalente es de $D_{e}=1.05 s=2.26 \mathrm{~m}$, lo que implica un factor de sustitución de:

$$
\left.a_{r}=\left(D_{c} / D_{e}\right)^{2}=0.9 / 2.26\right)^{2}=16 \%
$$

\section{ASIENTO SIN COLUMNAS}

Como paso previo al cálculo del asiento final con columnas mediante diferentes metodologías, se muestra aquí el cálculo del asiento final sin columnas en el centro del terraplén utilizando la teoría edométrica. Debido a la anchura del terraplén, se supone que la carga aplicada por el terraplén $(\Delta \sigma=6 \cdot 20=120 \mathrm{kPa})$ no se distribuye en profundidad y mantiene su valor constante.

El cálculo del asiento total $s_{z o}=21.1 \mathrm{~cm}$ se obtiene como suma de la deformación parcial de cada capa o estrato $(\Delta H)$ (Tabla 1). La deformación de cada capa se obtiene como producto de su espesor inicial $\left(H_{o}\right)$ y la deformación unitaria media de la capa $(\varepsilon)$. Para el relleno y las arenas, la deformación unitaria es directamente el cociente entre el incremento de carga provocado por el terraplén $(\Delta \sigma)$ y su módulo edométrico $\left(E_{m s}\right)$. Para los limos, se utiliza la teoría edométrica, teniendo

Tabla 1. Deformación de cada estrato para el caso sin columnas.

\begin{tabular}{|c|c|c|c|c|c|}
\hline Estrato & $\begin{array}{c}\Delta \sigma \\
(\mathbf{k P a})\end{array}$ & $\begin{array}{c}E_{m s} \\
\text { (MPa) }\end{array}$ & $\begin{array}{c}\varepsilon \\
(\%)\end{array}$ & $\begin{array}{c}H_{o} \\
(\mathbf{m})\end{array}$ & $\begin{array}{c}\Delta H \\
(\mathrm{~cm})\end{array}$ \\
\hline Relleno & 120 & 10.4 & 1.2 & 1 & 1.2 \\
\hline Limos & 120 & 3.1 & 3.8 & 4.5 & 17.1 \\
\hline Arenas & 120 & 14.8 & 0.8 & 3.5 & 2.8 \\
\hline
\end{tabular}

en cuenta que se suponen normalmente consolidados y que la tensión efectiva vertical en el punto medio $(-3.25 \mathrm{~m})$ es de $\sigma_{z o}^{\prime}=38 \mathrm{kPa}$ :

$$
\varepsilon=\frac{\Delta \mathrm{e}}{1+e_{\mathrm{o}}}=\frac{C_{C}}{1+e_{\mathrm{o}}} \log \frac{\sigma_{z \mathrm{O}}^{\prime}+\Delta \sigma}{\sigma_{z 0}^{\prime}}=3.8 \%
$$

El asiento del relleno y de las arenas se supone instantáneo y el asiento de los limos diferido en el tiempo.

\section{ASIENTO CON COLUMNAS}

\subsection{Guía de Cimentaciones en Obras de Carretera}

La Guía de Cimentaciones (9) proporciona, de forma simplificada, el valor del factor de reducción de asientos $(\beta)$ como función exclusivamente del factor de sustitución $\left(a_{r}\right)$. Esta relación se da de forma tabulada (9), pero obedece con gran aproximación a la siguiente expresión:

$$
\beta=\left(1-a_{r}\right)^{2}=(1-0.16)^{2}=0.7
$$

De esta forma, la deformación de la capa de limos se vería reducida al $70 \%$ por la presencia de las columnas $[2,6]$ $\left(s_{z}=17.1 \cdot 0.7=12 \mathrm{~cm}\right)$ y el asiento total es de $16 \mathrm{~cm}$.

La ROM 0.5-05 (10) utiliza la misma formulación que la propuesta por la Guía. Aquí se menciona por sencillez sólo la Guía (9), pero los comentarios también son válidos para la ROM (10). La formulación propuesta [6] es una media conservadora de otras metodologías de diseño comúnmente empleadas (p. ej., (11)) y se ha comprobado que constituye también una media conservadora de medidas en obras reales (18). La propia Guía indica que: «lógicamente, es de esperar que la reducción de asientos sea mayor cuando la columna es de mejor calidad. También sería de esperar que la deformabilidad del suelo jugase algún papel en este problema». Estos aspectos se tienen en cuenta en otras metodologías que se comentan a continuación. En la Guía no se tienen en cuenta por la falta de evidencias experimentales en obras reales, por ejemplo, las propiedades de las columnas no suelen conocerse y porque: «el procedimiento de estimación de la reducción de asientos no se encuentra aún completamente establecido».

En cuanto al cálculo del proceso de consolidación, se puede utilizar de forma simplificada y con una modificación que se detalla más adelante, la metodología propuesta para mechas drenantes. Esta metodología se basa en desacoplar la consolidación por flujo vertical y horizontal usando la ecuación de Carrillo (19):

$$
1-U=\left(1-U_{r}\right)\left(1-U_{z}\right)
$$

siendo $U_{r}$ y $U_{z}$ los grados de consolidación por flujo radial y vertical, respectivamente, y $U$ el grado de consolidación total.

Para la consolidación por flujo vertical, se puede utilizar la teoría de consolidación unidimensional de Terzaghi-Frölich:

$$
T_{v}=c_{v} t / H^{2}=0.043
$$




$$
U_{z}=\sqrt{4 T_{v} / \pi}=23.3 \%
$$

Para el cálculo del máximo camino de drenaje en dirección vertical, $H$ en [8], se ha tomado la mitad del espesor de la capa de limos (2.25 m), suponiendo que las arenas están conectadas hidráulicamente al nivel freático (N.F.) (Figura 6).

Para la consolidación por flujo radial, la formulación de la Guía es la propuesta por Barron (20):

$$
U_{r}=1-e^{\left.-8 c_{h} t / \mu D_{e}^{2}\right)}
$$

donde

$$
\mu=\ln \left(D_{e} / D_{c}\right)-0.75=0.17
$$

La ecuación [11] es la que recoge la Guía y es una simplificación de la expresión original propuesta por (20) para casos en los que $D_{e}>>D_{c}$, como ocurre habitualmente en las mechas drenantes (tratamiento de mejora al que hace referencia la Guía). Sin embargo, para columnas de grava, ello conlleva diferencias apreciables, y es necesario utilizar la expresión original:

$$
\mu=-\frac{\ln a_{r}}{2\left(1-a_{r}\right)}-\frac{3-a_{r}}{4}=0.38
$$

De esta forma, el grado de consolidación radial a los 50 días para la capa de limos es $U_{r}=83.0 \%[10,12]$ y el grado de consolidación total de la capa de limos es $U=86.9 \%$ [7], la deformación de la capa de limos es $10.4 \mathrm{~cm}$ y el asiento total al cabo de 50 días es de $14.4 \mathrm{~cm}$. Si se desea ser conservador y tener en cuenta la contaminación de la zona exterior de las columnas, se puede penalizar el diámetro de la columna (p. ej., un 5-10\% menos) para el análisis de la consolidación radial $[10,12]$.

\subsection{Método de Priebe}

Es el método más antiguo de cálculo de reducción de asientos (21) y probablemente el más utilizado en el mundo. Su éxito se debe a que es un método ampliamente contrastado con casos reales, a pesar de que su desarrollo teórico contiene varias hipótesis simplificadoras, tal y como se detalla en (22). El método de Priebe $(11,21)$ no estudia el proceso de consolidación como tal, aunque ello puede hacerse por algún otro método.

$\mathrm{Al}$ igual que en (9,10), el cálculo del asiento con columnas se realiza de un modo indirecto, es decir a través del asiento sin columnas y un factor de mejora, que es el inverso del factor de reducción de asientos. De esta forma, el método proporciona únicamente el factor de mejora $(n=1 / \beta)$.

La formulación original (21) proporciona un factor de mejora básico, que depende únicamente del factor de sustitución $\left(a_{r}\right)$, del ángulo de rozamiento de las columnas $\left(\varphi_{c}\right)$ y del coeficiente de Poisson del suelo $\left(v_{s}\right)$ :

$$
n_{\mathrm{o}}=1+a_{r}\left[\frac{0.5+f\left(v_{s}, a_{r}\right)}{K_{a c} f\left(v_{s}, a_{r}\right)}-1\right]
$$

donde

$$
f\left(v_{s}, a_{r}\right)=\frac{\left(1-v_{s}\right)\left(1-a_{r}\right)}{1-2 v_{s}+a_{r}}
$$

y

$$
K_{a c}=\tan ^{2}\left(45^{\circ}-\varphi_{c} / 2\right)
$$

La ecuación [13] se suelen proporcionar de forma gráfica para mayor sencillez de uso. Para $v_{s}=1 / 3$ y $a_{r}=0.16, f=1.13$. Por otra parte, tomando $\varphi_{c}=40^{\circ}$, se obtiene $K_{a c}^{r}=0.217 \mathrm{y}$ el factor de mejora básico es $n_{o}=1.90$. Se puede comprobar que ello proporciona un factor de reducción de asientos $(\beta=0.53)$ inferior (es decir, mayor mejora) al obtenido con la Guía $(\beta=0.7)$.

Uno de los inconvenientes del método original (21) es que para una mejora o sustitución total del suelo blando $\left(a_{r}=1\right)$ proporciona un factor de mejora infinito [13,14]. Para resolver este problema, Priebe (11) propuso una translación horizontal de la curva original [13], de tal forma que para $a_{r}=1$, el factor de mejora coincida con la relación de módulos confinados o edométricos $\left(E_{m c} / E_{m s}\right)$. De esta forma, el factor de mejora $\left(n_{1}\right)$ debe obtenerse a partir de [13], pero utilizando un factor de sustitución modificado (11). En este caso $E_{m c}=44.4$ $\mathrm{MPa}$, tomando $E_{m s}=3.1$ (Tabla 1 ) y $v_{s}=1 / 3$, se obtiene que $n_{1}=1.84$.

La corrección anterior provoca siempre una minoración del factor de mejora. La otra corrección, en este caso una mayoración, que Priebe (11) planteó al método original (21) es la consideración de las tensiones iniciales en el suelo y la columna. Para ello, se debe corregir el factor de mejora $n_{1}$ por un factor $f_{d} \geq 1$ (11).

Teniendo en cuenta los datos del ejercicio planteado, $S C F=6.6, \Delta \sigma_{z c}=428 \mathrm{kPa}$ y $f_{d}=1.2$. Por último, Priebe (11) establece una comprobación o valor máximo para este factor, que no se excede en este ejemplo de cálculo. Así, el factor de mejora final es $n_{2}=2.2$, lo que supone un factor de reducción de asientos $\beta=0.45$, una deformación del estrato de limos de $7.7 \mathrm{~cm}$, y un asiento final de $11.7 \mathrm{~cm}$.

Debido a que las dos correcciones introducidas por Priebe (11) complican el cálculo y se compensan o suelen proporcionar valores inferiores del asiento, en muchos casos se utiliza únicamente el factor básico de mejora, $n_{0}$.

\subsection{Castro y Sagaseta (2009)}

Existe un grupo de soluciones que analizan de forma teórica el comportamiento de la celda unidad, imponiendo las condiciones de equilibrio (equilibrio de las tensiones verticales y equilibrio de las tensiones en el contacto radial suelo-columna) y de compatibilidad de deformaciones (asiento uniforme y compatibilidad del desplazamiento radial en el contacto suelo-columna). En la Tabla 2, se resumen las principales capacidades de predicción e hipótesis de estas soluciones.

La solución de Balaam y Booker (23) constituye la solución exacta para los asientos a corto y largo plazo suponiendo un comportamiento elástico lineal de la columna y del suelo circundante. Para compensar la hipótesis optimista de com- 
Tabla 2. Capacidades predictivas e hipótesis de las principales soluciones analíticas.

\begin{tabular}{|c|c|c|c|c|c|c|c|}
\hline & \multirow[b]{2}{*}{ Asiento } & \multirow[b]{2}{*}{ Consol. } & \multirow[b]{2}{*}{ Ensacadas } & \multicolumn{3}{|c|}{ Comportamiento columna } & \multirow[b]{2}{*}{$\boldsymbol{\tau} \neq \mathbf{O}$} \\
\hline & & & & Elástica & E-P simple & E-P & \\
\hline Balaam y Booker (1981) (23) & $\checkmark$ & & & $\checkmark$ & & & \\
\hline Balaam y Booker (1985) (24) & $\checkmark$ & & & & & $\checkmark$ & 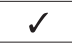 \\
\hline Castro y Sagaseta (2009) (25) & $\checkmark$ & 4 & & & $\checkmark$ & & \\
\hline Castro y Sagaseta (2011) (26) & $\checkmark$ & 2 & 2 & & $\checkmark$ & & \\
\hline Pulko et al. (2011) (27) & $\checkmark$ & & $\checkmark$ & & & $\checkmark$ & \\
\hline Castro y Sagaseta (2013) (28) & $\checkmark$ & $\checkmark$ & $\checkmark$ & & & $\checkmark$ & \\
\hline Pulko y Logar (2016) (29) & $\checkmark$ & $\checkmark$ & & & & $\checkmark$ & \\
\hline Pulko y Logar (2017) (30) & $\checkmark$ & $\checkmark$ & $\checkmark$ & & & 2 & \\
\hline
\end{tabular}

portamiento elástico de la columna, es necesario utilizar módulos conservadores para la columna. Para el proceso de consolidación, presentan un análisis por elementos finitos. Estos autores desarrollaron posteriormente una solución analítica teniendo en cuenta la plastificación de la columna (24). Esta solución no es utilizada habitualmente porque presenta una formulación analítica compleja, al no despreciar las tensiones tangenciales en el contacto columna-suelo, y requiere solución numérica.

Castro y Sagaseta (25) presentaron una solución analítica que permite estudiar simultáneamente el proceso de consolidación y las deformaciones en la columna y el suelo circundante. Esta solución estudia de forma independiente el comportamiento de las diferentes rebanadas de columnas de espesor despreciable a diferentes profundidades, lo que es equivalente a despreciar las tensiones tangenciales. Esta solución se puede extender fácilmente al caso de columnas ensacadas (26). Además, en estas dos soluciones $(25,26)$ se desprecian las deformaciones elásticas en la columna durante su deformación plástica (hipótesis denominada «E-P simple» en la Tabla 2). Se puede comprobar que esta última hipótesis es adecuada para columnas convencionales sin ensacar pero que, para columnas ensacadas, despreciar estas deformaciones elásticas produce diferencias apreciables (28).

Por otra parte, Pulko et al. (27) presentaron la solución drenada para la situación final a largo plazo, que proporciona el mismo resultado que la propuesta por Castro y Sagaseta (28) para la situación final, y es prácticamente idéntica a Castro y Sagaseta (25) para el caso de columnas sin ensacar. Finalmente, Pulko y Logar $(29,30)$ han desarrollado soluciones analíticas acopladas, tanto para columnas ensacadas como sin ensacar, para estudiar el proceso de consolidación (solución poroelástica en la que el suelo no es infinitamente incompresible). Para grados de consolidación bajos, estas soluciones poroelásticas predicen de forma correcta una mayor rapidez en el proceso de consolidación, pero para grados de consolidación como los que suelen ser de interés (70-100 \%) proporcionan resultados similares a las soluciones desacopladas $(25,28)$.

De esta forma, para el caso a estudiar, se utilizará la solución (25). En caso de que sólo fuera necesario estudiar el asiento final, sería suficiente utilizar (27). Estas dos soluciones se pueden utilizar de forma sencilla a través de hojas de cálculo. Por simplicidad y para evitar repeticiones, no se presenta aquí el detalle de la formulación de estas soluciones.

Los datos de entrada para este caso son los mostrados en la Figura 6 con los siguientes matices:
1. Por sencillez, para obtener los resultados se toma el punto medio del estrato limoso como representativo, sin realizar la integración exacta a diferentes profundidades.

2. La solución considera el efecto del coeficiente de empuje en reposo del suelo blando $\left(K_{\mathrm{os}}\right)$, ya que cuanto mayor es su valor, mejor confinada lateralmente está la columna. En este caso, su valor se ha obtenido en base al ángulo de rozamiento $\left(\varphi_{s}=25^{\circ}\right)$, utilizando la fórmula de Jaky $\left(K_{0}=1-\operatorname{sen} \varphi\right)$.

3. El módulo edométrico del suelo se ha ajustado al rango de tensiones correspondiente. El módulo edométrico obtenido en la Tabla 1 para los limos $\left(E_{m s}=3.1 \mathrm{MPa}\right)$ corresponde al rango de tensiones en el caso de que los limos no se mejoren (de $38 \mathrm{kPa}$ a $38+120 \mathrm{kPa}$ ). Sin embargo, cuando el suelo se mejora con columnas de grava, la tensión vertical final soportada por los limos es menor, de tal forma, que el módulo edométrico medio para ese intervalo tensional es también menor. De esta forma, se requiere un cálculo iterativo del módulo. Por ejemplo, en este caso se estimó la variación tensional en el suelo con un módulo de 3.1 $\mathrm{MPa}$, proporcionando un incremento tensional en el suelo de $\Delta \sigma_{z s}=74 \mathrm{kPa}$ y un módulo edométrico medio de $E_{m s}=2.5 \mathrm{MPa}[16]$. Con este nuevo módulo, se puede comprobar que el incremento prácticamente no varía y, por tanto, se toma como válido. Se puede comprobar que las diferencias no son despreciables (2.5 frente a 3.1 MPa). En un sentido estricto, el suelo deja de estar en condiciones edométricas (confinado lateralmente) con la presencia de las columnas y sería necesario realizar el análisis en función de la tensión media (31), pero desde un punto de vista práctico se considera suficientemente aproximado utilizar la tensión vertical, sin considerar la tensión radial para obtener el módulo medio.

$$
\frac{1}{E_{m s}}=\frac{\varepsilon}{\Delta \sigma_{z s}}=\frac{C_{C}}{\Delta \sigma_{z s}\left(1+e_{0}\right)} \log \frac{\sigma_{z s o}^{\prime}+\Delta \sigma_{z s}}{\sigma_{z s o}^{\prime}}
$$

Esta solución proporciona directamente el asiento con columnas $\left(s_{z}=11.9 \mathrm{~cm}\right)$, que corresponde a la deformación de los limos. Así, el asiento total es de $15.9 \mathrm{~cm}$. El resultado es casi coincidente con el predicho por la Guía de Cimentaciones para este caso concreto, lo que parece deberse a unos parámetros conservadores de las columnas de grava (Figura 6) y a una tensión inicial media del estrato de limos baja $\left(\sigma_{z s 0}^{\prime}=38 \mathrm{kPa}\right)$, pero no significa que no pueda haber diferencias en otros casos, en función de la configuración geométrica y elección de los parámetros.

En cuanto al asiento alcanzado al cabo de 50 días, su valor es de $10.3 \mathrm{~cm}$, tomando el punto medio como representativo y 


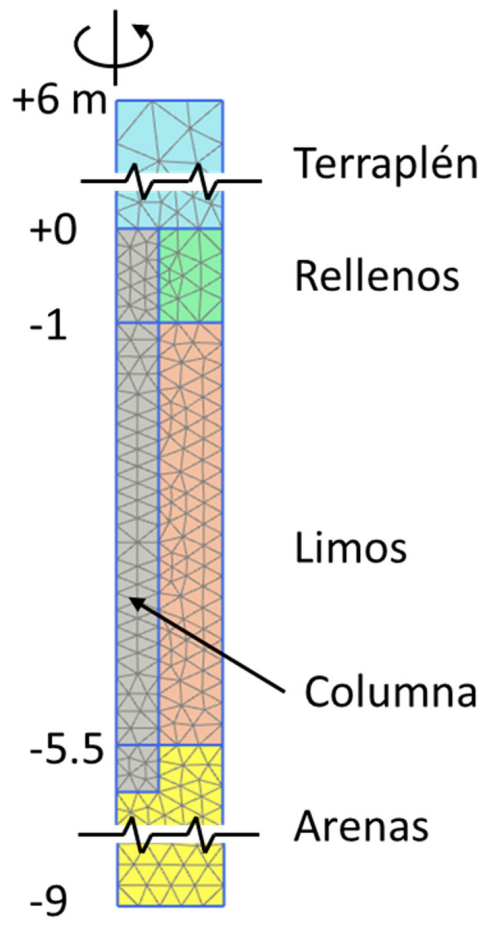

Figura 7. Malla de elementos finitos para el caso de estudio.

teniendo en cuenta el drenaje en dirección vertical a través de la fórmula de Carillo (19) [7]. Esto supone un $86 \%$ de la deformación final.

\subsection{Elementos finitos}

La simulación mediante elementos finitos se ha llevado a cabo mediante el código comercial Plaxis 2D 2017 (32). La geometría del problema y la malla elegida se muestran en la Figura 7. El problema se analiza en simetría axial, utilizando la «celda unidad». Las condiciones de contorno son: contorno inferior fijo, contornos laterales fijos únicamente en dirección horizontal y contorno superior libre. Además, los contornos laterales se consideran impermeables.

Los niveles de terraplén, rellenos, arenas y la columna de grava se representan mediante un modelo elástico lineal, plástico perfecto con una ley de flujo no asociada (modelo denominado «Mohr-Coulomb» en Plaxis). Los parámetros necesarios para este modelo son los indicados en la Figura 6. Para el terraplén, rellenos y arenas, la dilatancia y la cohesión se consideran nulas, aunque para esta última, se toma un valor ligeramente superior a o $(c=0.1 \mathrm{kPa}$ en este caso) para evitar problemas numéricos.

En cuanto al nivel de limos, existe la posibilidad de utilizar el modelo «Mohr-Coulomb» (M-C) tomando el módulo edométrico medio del apartado anterior $\left(E_{m s}=2.5 \mathrm{MPa}\right)$. Esto se va a realizar para comparar con la solución analítica anterior, pero también se puede utilizar un modelo más avanzado que permita utilizar directamente parámetros de la teoría edométrica $\left(C_{C}\right)$ para los limos. En este sentido, existen fundamentalmente 3 modelos en este código comercial: modelo "Modified Cam Clay» (MCC), modelo «Soft Soil» (SS) y modelo «Hardening Soil» (HS).

El modelo MCC es riguroso desde un punto de vista teórico (p. ej., (33)) y adecuado para este tipo de situaciones. Sin em- bargo, los códigos comerciales desaconsejan su uso de forma general y proponen modelos alternativos debido, fundamentalmente, a los siguientes motivos:

1. Fuera del plano de compresión triaxial, considera la superficie de rotura de Drucker-Prager, en lugar de MohrCoulomb, lo que implica una sobreestimación de la resistencia en trayectorias de deformación plana o extensión triaxial (p. ej., (34)).

2. En suelos sobreconsolidados («lado seco»), el modelo puede predecir reblandecimiento, lo que puede ocasionar problemas numéricos (p. ej., dependencia de la malla).

3. Para trayectorias de compresión confinada (edométrica), sobreestima el coeficiente de empuje en reposo $K_{0}$. Esto se debe a la forma del potencial plástico, que coincide con la superficie de plastificación, ya que se supone una ley de flujo asociada.

El modelo SS (32) se basa en el modelo MCC pero presenta modificaciones para evitar los 3 «problemas» mencionados del modelo MCC:

1. Utiliza la superficie de rotura de Mohr-Coulomb (c, $\varphi$ ).

2. Limita la superficie de plastificación elíptica con el criterio de Mohr-Coulomb para evitar «reblandecimiento».

3. Independiza la forma de la superficie de plastificación («cap») de la pendiente de la recta de rotura, de tal forma que ésta se ajusta para reproducir adecuadamente el coeficiente de empuje en reposo, bien el introducido de forma manual por el usuario o el calculado por defecto utilizando la fórmula de Jaky.

El modelo SS resulta adecuado para suelos blandos en situaciones de carga, como la del caso de estudio. Conviene destacar que el cálculo de las deformaciones volumétricas en el modelo SS es ligeramente diferente al edométrico tradicional (usado por ejemplo en el modelo MCC) al considerar cohesión:

$$
\begin{array}{cc}
\varepsilon_{v}=\lambda^{*} \ln \left(p^{\prime} / p_{\mathrm{o}}^{\prime}\right) & \text { Modelo MCC } \\
\varepsilon_{v}=\lambda^{*} \ln \left(\frac{p^{\prime}+c \cdot \cot \varphi}{p_{\mathrm{o}}^{\prime}+c \cdot \cot \varphi}\right) & \text { Modelo SS }
\end{array}
$$

para procesos de carga noval (suelo normalmente consolidado), donde $p$ es la tensión media y

$$
\lambda^{*}=C_{C} /\left[\left(1+e_{0}\right) \ln 10\right]
$$

$\lambda^{*}$ es el índice de compresión noval en escala de logaritmos neperianos para la tensión efectiva y normalizado por el volumen específico (la unidad más el índice de poros inicial) [19].

Por último, el modelo HS (32) es un modelo más complejo y avanzado que permite incluir fundamentalmente los dos aspectos siguientes:

1. Permite considerar una dependencia exponencial de los módulos de elasticidad con la tensión media. De esta forma, el exponente $m$ puede variar entre un valor de o (valor constante de los módulos, elasticidad lineal) y un valor de 1 (dependencia lineal del módulo con la tensión media, modelo edométrico). 
2. Considera dos superficies de plastificación con rigidización independientes: una para trayectorias de compresión (deformaciones fundamentalmente volumétricas) y otra para trayectorias de corte (deformaciones fundamentalmente desviadoras). Esto permite reproducir una ley hiperbólica en las trayectorias de corte (p. ej., compresión triaxial).

Para el caso de estudio, la deformación de la capa de limos, predicha por los diferentes modelos, se resume en la Tabla 3, tanto para el caso con columnas como sin columnas. Para los niveles de relleno y de arenas, sus deformaciones son las mismas que las calculadas analíticamente para el caso sin columnas (Tabla 1), y ligeramente inferiores para el caso con columnas (del orden de 0.8 y $2.6 \mathrm{~cm}$, respectivamente). Para el caso con columnas, el asiento no es perfectamente uniforme y encima de la columna el asiento total es del orden de 0.5 $\mathrm{cm}$ menor que en el punto medio entre columnas, ubicación a la que corresponden los valores de deformaciones indicados (Tabla 3).

En cuanto a los diferentes valores de la deformación de la capa de limos sin columnas en función del modelo constitutivo empleado (Tabla 3), cabe comentar lo siguiente:

- El valor proporcionado por el modelo M-C $(17.1 \mathrm{~cm})$ coincide con el cálculo analítico sencillo $\left(\varepsilon=\Delta \sigma / E_{m s}\right)$. Tal y como se realizó para la solución analítica (25) es necesario ajustar un valor de $E_{m s}$ para el caso sin columnas y otro valor de $E_{m s}$ para el caso con columnas debido al diferente incremento tensional en los limos.

- El valor proporcionado por el modelo MCC $(17.7 \mathrm{~cm})$ corresponde al modelo edométrico [5]. Las diferencias con el cálculo analítico $(17.1 \mathrm{~cm}$, Tabla 1$)$ se deben a que en el cálculo analítico se tomó como representativo el punto medio del estrato y su tensión efectiva vertical inicial $\left(\sigma_{z s o}^{\prime}=38\right.$ $\mathrm{kPa}$ ). Si se divide el estrato de limos en varias capas para el cálculo analítico, el valor calculado tiende al obtenido numéricamente.

- El valor obtenido con el modelo SS coincide estrictamente con el modelo edométrico (MCC) $(17.7 \mathrm{~cm})$ cuando el valor de la cohesión es nulo ( $\mathrm{SS}^{*}$ ). En caso de utilizar el valor de $c=10 \mathrm{kPa}$ (SS), el asiento es ligeramente inferior debido a que en el modelo tiene un efecto equivalente a incrementar el valor de la tensión efectiva inicial para el cálculo de las deformaciones volumétricas [18].

- Los resultados proporcionados por el modelo HS no son estrictamente iguales a los proporcionados por el modelo edométrico debido a la formulación del modelo y la influencia de la superficie de plastificación para trayectorias de corte. Además, la influencia de introducir un valor de la cohesión no nulo es más notable que para el modelo SS.
En cuanto al factor de reducción de asientos, $\beta$, está entre 0.57 y 0.71 (Tabla 3). El valor más adecuado se considera el proporcionado por el modelo $\mathrm{SS}^{*}(\beta=0.68)$. El modelo MCC proporciona un valor ligeramente inferior $(\beta=0.64)$ al del modelo $\mathrm{SS}^{*}$, es decir, mayor mejora, debido a la sobreestimación del coeficiente de empuje en reposo $\left(K_{\mathrm{os}}=0.74\right)$, frente al proporcionado en los otros modelos $\left(K_{\mathrm{os}}=0.58\right)$. Esto hace que la columna esté algo mejor confinada lateralmente y reduzca algo más el asiento.

Respecto a la evolución de los asientos con el tiempo, es necesario calcular el valor de la permeabilidad horizontal y vertical. Estas permeabilidades se han calculado en base al módulo edométrico medio con columna $\left(E_{m s}=2.5 \mathrm{MPa}\right)$ :

$$
c_{v}=E_{m} k_{z} / \gamma_{w}
$$

Así, los valores resultantes son $k_{H}=4 \cdot 10^{-10} \mathrm{~m} / \mathrm{s}$ y $k_{V}=2 \cdot 10^{-10} \mathrm{~m} / \mathrm{s}$. Para el resto de suelos se ha supuesto un valor suficientemente alto como para que no se generen excesos de presión intersticial en los mismos $\left(k=10^{-5} \mathrm{~m} / \mathrm{s}\right)$.

El valor de la deformación de la capa de limos al cabo de 50 días para los distintos modelos constitutivos empleados se resume en la Tabla 3. Además, también se presenta ese valor en porcentaje respecto a la deformación final con columnas. Se puede observar que los resultados son similares, en torno al 90\%. Para analizar más en detalle el proceso de consolidación, se muestra la deformación en función del tiempo en la Figura 8 para los principales modelos constitutivos (M-C, MCC, SS $^{*}$ ). La principal diferencia entre el modelo MCC y $\mathrm{SS}^{*}$ es el diferente valor de $K_{\mathrm{os}}$, lo que hace que la columna esté mejor confinada en el primero y alcance un estado de plastificación triaxial algo más tarde (proporcionando un valor del asiento final algo menor). En cuanto al modelo M-C, la principal diferencia es que al no considerar la no-linealidad de los limos, subestima ligeramente el ritmo de deformación en la primera mitad y lo sobreestima en la segunda mitad, proporcionando un valor compensado para la deformación final.

\section{DISCUSIÓN Y COMPARACIÓN DE RESULTADOS}

El objetivo del presente caso de estudio ha sido analizar las opciones y diferencias entre los diferentes métodos de cálculo, sin tener en cuenta las posibles diferencias derivadas de la interpretación de ensayos de campo y laboratorio.

Un resumen de los valores predichos por los diferentes métodos para el ejemplo de cálculo propuesto se muestra en la Tabla 4. Hay que recordar que el valor del asiento sin colum-

Tabla 3. Deformación de la capa de limos predicha por los diferentes modelos constitutivos.

\begin{tabular}{|l|c|c|c|c|c|}
\hline \multicolumn{1}{|c|}{ Deformación (cm) } & M-C & MCC & SS & SS $^{*}$ & HS \\
\hline Sin columnas (final) & $17.1 / 21.2^{* *}$ & 17.7 & 17.0 & 17.7 & 14.0 \\
\hline Con columnas (final) & 12.1 & 11.4 & 11.6 & 12.1 & 9.2 \\
\hline Reducción asiento, $\beta$ & $0.71 / 0.57$ & 0.64 & 0.68 & 0.68 & 0.66 \\
\hline Con columnas (50 días) & 10.3 & 10.5 & 10.7 & 11.0 & 0.63 \\
\hline \% deformación (50 días) & $85 \%$ & $92 \%$ & $92 \%$ & $91 \%$ & $97 \%$ \\
\hline
\end{tabular}

* $c=0.1 \mathrm{kPa}$

** Deformación con $E_{m s}=3.1 \mathrm{MPa}$ y $E_{m s}=2.5 \mathrm{MPa}$ 


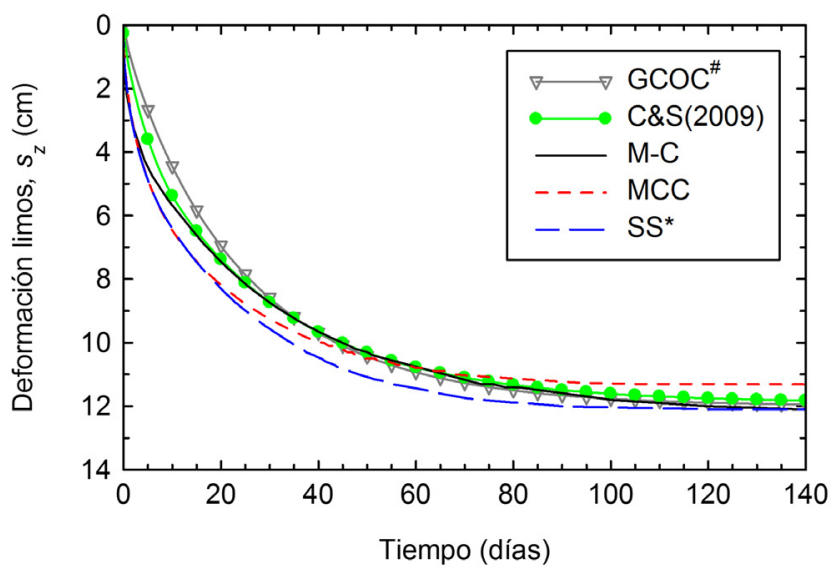

Figura 8. Evolución del asiento con el tiempo para diferentes métodos.

nas debería ser unívocamente $17.7 \mathrm{~cm}$, pero que para algunos casos se ha obtenido $17.1 \mathrm{~cm}$ al tomar el punto medio como representativo [5] (Tabla 1) y no dividir la capa de limos en varios estratos. En la Figura 9 también se presenta una comparación del factor de reducción de asientos predicho por Priebe (21) y la Guía de Cimentaciones (GCOC) (9). Se puede comprobar que, de forma aproximada, la GCOC proporciona un valor conservador y Priebe (21) guarda una mayor correlación con los datos de campo. Los datos de campo mostrados en la Figura 9 se han tomado de la recopilación bibliográfica realizada en (35). En la Figura 9 también se presentan las curvas que indican el valor del factor de concentración de tensiones $(S C F)$ que corresponde a cada pareja de valores $\left(a_{r}, \beta\right)$, aplicando equilibrio de tensiones verticales y suponiendo que la reducción de asientos es proporcional a la reducción de tensiones en el suelo blando:

$$
1 / \beta=p_{a} / \sigma_{z s}=1-a_{r}+a_{r} S C F
$$

Si se considera un comportamiento edométrico del suelo blando mejorado con columnas, la tensión inicial y el incremento de tensión influyen en el valor obtenido del factor de concentración de tensiones, $S C F$. En la Figura 9 se puede comprobar que valores del factor de sustitución superiores a 0.5 no son habituales y, además, las expresiones de la GCOC (9) y de Priebe (21) no proporcionan un buen ajuste en ese rango.

En cuanto a la evolución de los asientos con el tiempo, Castro y Sagaseta (36) mostraron que el método de Barron (20) subestima de forma general la velocidad de los asientos para columnas de grava, mientras que el método de Castro y Sagaseta (25) suele proporcionar valores adecuados para grados de consolidación superiores al 60\%. En el presente caso (Fi-

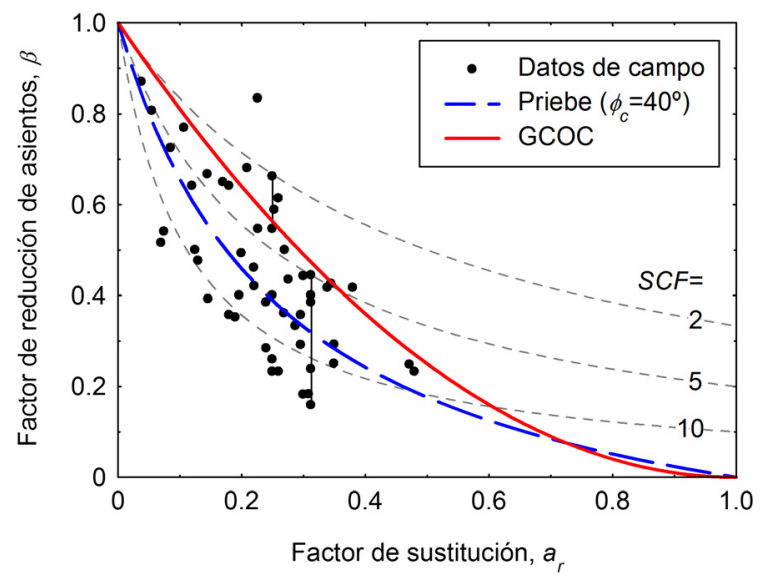

Figura 9. Factor de reducción de asientos: comparación de métodos analíticos y medidas en casos reales.

gura 8), las diferencias son despreciables respecto a los análisis numéricos para tiempos superiores a 40 días.

Para el caso propuesto (Tabla 4), la reducción de asiento obtenida por elementos finitos (EF) utilizando modelos edométricos para la capa de limos (MCC y SS*) es ligeramente inferior a la dada por la GCOC. Para los casos de un modelo elástico lineal plástico perfecto (C\&S(2009) (25) y EF-MC), el asiento con columnas es similar al dado por la GCOC y al obtenido con los otros análisis por EF; sin embargo, el valor del factor de reducción de asientos puede variar de forma notable en función de si se calcula con el mismo módulo edométrico o se distinguen dos módulos diferentes para el caso con columnas y sin columnas debido a los diferentes incrementos tensionales.

En el caso estudiado los valores propuestos por la GCOC concuerdan de forma razonable con los otros métodos (EF y C\&S(2009) (25)), exceptuando la solución de Priebe (21). Sin embargo, cabe analizar que el valor de los parámetros utilizados para las columnas (Figura 6) en los análisis por EF y en Castro y Sagaseta (25) son conservadores y si se utilizaran otros parámetros (p. ej., teniendo en cuenta una mayor resistencia y rigidez de las columnas o un mayor coeficiente de empuje debido a los efectos de la instalación de las columnas), el asiento sería más similar al proporcionado por Priebe (21).

Por último, indicar que en todos los cálculos realizados se ha supuesto la hipótesis de pequeñas deformaciones, es decir, no se ha tenido en cuenta el asiento adicional ocasionado por el material de relleno de terraplén que es necesario añadir para compensar el asiento producido y alcanzar la cota superior de terraplén deseada.

Tabla 4. Deformación de la capa de limos predicha por los diferentes métodos.

\begin{tabular}{|c|c|c|c|c|c|c|}
\hline Deformación (cm) & GCOC\# & Priebe & C\&S(2009) & EF-MC & EF-MCC & EF-SS* \\
\hline Sin columnas (final) & 17.1 & 17.1 & $17.1 / 21.2^{* *}$ & $17.1 / 21.2^{* *}$ & 17.7 & 17.7 \\
\hline Con columnas (final) & 12.0 & 9.1 & 11.9 & 12.1 & 11.4 & 12.1 \\
\hline Reducción asiento, $\beta$ & 0.70 & 0.53 & $0.70 / 0.56$ & $0.71 / 0.57$ & 0.64 & 0.68 \\
\hline Con columnas (50 días) & 10.4 & - & 10.3 & 10.3 & 10.5 & 11.0 \\
\hline \% deformación (5o días) & $87 \%$ & - & $87 \%$ & $85 \%$ & $92 \%$ & $91 \%$ \\
\hline
\end{tabular}

* $c=0.1 \mathrm{kPa}$

** Deformación con $E_{m s}=3.1 \mathrm{MPa}$ y $E_{m s}=2.5 \mathrm{MPa}$

${ }^{*}$ Usando la solución de Barron (20) [10,12] 


\section{CONCLUSIONES}

Este artículo recoge algunos aspectos teóricos recientes relativos a columnas de grava que pueden resultar útiles para su diseño y cálculo. Se ha presentado un ejemplo de cálculo utilizando diferentes métodos semi-empíricos, analíticos y numéricos. Cabe destacar que, de forma general, éstos métodos han proporcionado resultados adecuados si se tienen en cuenta las limitaciones y posibilidades de cada una de las metodologías. En concreto, se derivan las siguientes conclusiones para cada uno de ellos:

- El método propuesto por la GCOC (9) es sencillo y suele proporcionar valores conservadores. Sólo considera el factor de sustitución, no permitiendo considerar la influencia de otros factores. Para el análisis del proceso de consolidación radial es necesario utilizar [10,12], que también suelen proporcionar valores conservadores.
- El método de Priebe (21) es más optimista, por lo que debe utilizarse con valores conservadores (p. ej., $\varphi_{c}=40^{\circ}$ ) y garantizar una correcta ejecución de las columnas (para que, por ejemplo, el ángulo de rozamiento de la columna sea superior al supuesto).

- El método de Castro y Sagaseta (25) permite tener en cuenta la influencia de diferentes factores como, por ejemplo, $\varphi_{c}, K_{o}, E_{c}, E_{m s}$. Sin embargo, es necesario una adecuada selección de los mismos, por ejemplo, ajustar el valor de $E_{m s}$ al rango de tensiones correspondiente.

- Los cálculos por elementos finitos proporcionan una gran flexibilidad en el análisis, pero es necesaria una adecuada comprensión de las hipótesis, modelos constitutivos y parámetros empleados (incluidos los utilizados por defecto por el código numérico). En el presente caso, por ejemplo, se ha observado la conveniencia de tomar la cohesión de los limos como nula cuando se utiliza el modelo SS o la ligera sobreestimación del coeficiente de empuje en reposo y, por tanto, de la reducción de asientos cuando se utiliza el modelo MCC.

\section{REFERENCIAS}

(1) Castro, J. Avances en el diseño y cálculo de columnas de grava. Parte II: Grupos de columnas y consolidación secundaria. Informes de la Construcción (aceptado en espera de publicación).

(2) Barksdale, R.D. y Bachus, R.C. (1983). Design and Construction of Stone Columns, Report FHWA/RD-83/o26. Springfield, Virginia: National Technical Information Service.

(3) Almeida, M., Riccio, M., Hosseinpour, I. y Alexiew, D. (2019). Geosynthetic Encased Stone Columns for Soft Soil Improvement. Leiden: CRC Press. https://doi.org/10.1201/9781315177144

(4) Aboshi, H., Ichimoto, E., Enoki, M. y Harada, K. (1979). The compozer - a method to improve characteristics of soft clay by inclusión of large diameter sand columns. Proc. Int. Conf. Soil Reinforcements: Reinforced Earth and Other Techniques, Paris, Vol. 1, pp. 211-216.

(5) Watts, K.S, Johnson, D., Wood, L.A. y Saadi, A. (2000). An in-strumented trial of vibro ground treatment supporting strip foundations in a variable fill. Géotechnique, 50(6): 699-708. https://doi.org/10.1680/geot.2000.50.6.699

(6) McCabe, B.A., Nimmons, G.J. y Egan, D. (2009). A review of field performance of stone columns in soft soils. Proc. ICE - Geotechnical Engineering, 162(6): 323-334. https://doi.org/10.168o/geng.2009.162.6.323

(7) Castro, J. y Karstunen, M. (2010). Numerical simulations of stone column installation. Canadian Geotechnical Journal, 47(10): 1127-1138. https://doi.org/10.1139/T10-019

(8) Serridge, C.J. (2015). Briefing: Dry bottom-feed stone column construction in soft fine-grained soils. Proc. ICE - Ground Improvement, 168: 159-161. https://doi.org/10.1680/grim.14.00019

(9) Ministerio de Fomento. (2009). Guía de Cimentaciones en Obras de Carretera. Ministerio de Fomento. Serie Monografías.

(10) Ministerio de Fomento. (2005). Recomendaciones Geotécnicas para Obras Marítimas y Portuarias. Ed. Puertos del Estado.

(11) Priebe, H.J. (1995). The design of vibro replacement. Ground Engineering, 28(10): 31-37.

(12) Mestat, Ph., Dhouib, A., Magnan, J.P. y Canépa, Y. (2004). Résultats de l'exercice de prévision des tassements d'un remblai construit sur des colonnes ballastées. International Symposium on Ground Improvement (ASEP-GI 2004). Paris, 9-10 Septiembre 2004. Paris: Presses de l'ENPC/LCPC.Vol. II, pp. 599-612.

(13) Mestat, Ph., Magnan, J.P. y Dhouib, A. (2006). Results of the settlement prediction exercise of an embankment founded on soil improved by stone columns. Proc. $6^{\text {th }}$ Eur. Conf. Num. Meth. Geotech. Eng. (NUMGE o6). Graz, Austria. pp. 471-476.

(14) Wehr, J. y Herle, I. (2006). Exercise on calculation of stone columns - Priebe method and FEM. Proc. $6^{\text {th }}$ European Conference on Numerical Methods in Geotechnical Engineering (NUMGE 06). Graz, Austria. pp. 773-776.

(15) Lambe, T.W. (1973). Predictions in soil engineering. Géotechnique, 23(2): 149-202. https://doi.org/10.1680/ geot.1973.23.2.151

(16) Bohn, C., Frank, R. y Lambert, S. (2013). Foundation settlement calculations with the pressuremeter method compared to other methods and resulting correlations. Proc. $18^{\text {th }}$ Int. Conf. Soil Mech. Geotech. Eng. Paris. Parallel session ISP 6.

(17) AFNOR. (2013). Eurocode 7 Part 1, French version NF EN 1997-1, Calcul géotechnique - Règles générales. Application standards for shallow (NF P 94-261, 2013) and pile foundations (NF P 94-262, 2013).

(18) Sagaseta, C. (2011). Cálculo de columnas de grava: algunos puntos oscuros. Ingeniería Civil, 162: 43-60.

(19) Carrillo, N. (1942). Simple two- and three-dimensional cases in the theory of consolidation. J. Math. Phys., 21: 1-5. https://doi.org/10.1002/sapm19422111

(20) Barron, R.A. (1948). Consolidation of fine-grained soils by drain wells. Trans. ASCE 113: 718-742.

(21) Priebe, H.J. (1976). Abschatzung des Setzungsverhaltens eines dursch Stopverdichtung Verbesserten Baugrundes. Die Bautechnik 53(5), 160-162. Traducido al castellano como "Estimación de los asientos de un terreno consolidado con columnas de grava”, Boletín de información del Laboratorio de Transporte y Mecánica del Suelo nº 116. 
(22) Sagaseta, C. (2006). Avances en el diseño de las técnicas de mejora del terreno. 6ª Sesión Jornadas Técnicas SEMSIGAETESS. Madrid: AETESS. pp. 13-26.

(23) Balaam, N.P. y Booker, J.R. (1981). Analysis of rigid rafts supported by granular piles. International Journal for Numerical and Analytical Methods in Geomechanics, 5: 379-403. https://doi.org/10.1002/nag.1610050405

(24) Balaam, N.P. y Booker, J.R. (1985). Effect of stone column yield on settlement of rigid foundations in stabilized clay. International Journal for Numerical and Analytical Methods in Geomechanics, 9: 331-351. https://doi.org/10.1002/ nag.1610090404

(25) Castro, J. y Sagaseta, C. (2009). Consolidation around stone columns. Influence of column deformation. International Journal for Numerical and Analytical Methods in Geomechanics, 33 (7): 851-877. https://doi.org/10.1002/nag.745

(26) Castro, J. y Sagaseta, C. (2011). Deformation and consolidation around encased stone columns. Geotextiles and Geomembranes, 29: 268-276. https://doi.org/10.1016/j.geotexmem.2010.12.001

(27) Pulko, B., Majes, B. y Logar, J. (2011). Geosynthetic-encased stone columns: Analytical calculation model. Geotextiles and Geomembranes, 29: 29-39. https://doi.org/10.1016/j.geotexmem.2010.06.005

(28) Castro, J. y Sagaseta, C. (2013). Influence of elastic strains during plastic deformation of encased stone columns. Geotextiles and Geomembranes, 37: 45-53. https://doi.org/10.1016/j.geotexmem.2013.01.005

(29) Pulko, B. y Logar, J. (2016). Fully coupled solution for the consolidation of poroelastic soil around elastoplastic stone columns. Acta Geotechnica, 12: 869-882. https://doi.org/10.1007/s11440-016-0484-2

(30) Pulko, B. y Logar, J. (2017). Fully coupled solution for the consolidation of poroelastic soil around geosynthetic encased stone columns. Geotextiles and Geomembranes, 45: 616-626. https://doi.org/10.1016/j.geotexmem.2017.08.003

(31) Castro, J., Cimentada, A., Da Costa, A., Cañizal, J. y Sagaseta, C. (2013). Consolidation and deformation around stone columns: Comparison of theoretical and laboratory results. Computers and Geotechnics, 49: 326-337. https://doi. org/10.1016/j.compgeo.2012.09.004

(32) Brinkgreve, R.B.J., Kumarswamy, S., Swolfs, W.M. y Foria, F. (2017). Plaxis 2017 User Manuals. Delft: Plaxis bv.

(33) Roscoe, K.H. y Burland, J.B. (1968). On the generalised stress-strain behaviour of 'wet' clay. In Engineering Plasticity (Heyman, J. y Leckie, F.A., eds.), pp. 563-609. Cambridge: Cambridge University Press.

(34) Potts, D.M. y Zdravkovic, L. (2000) Some pitfalls when using Modified Cam Clay. Soil-Structure interaction in Urban Civil Engineering. Editors: Avdelas. Publisher: European Commision.

(35) Castro, J. (2008). Análisis teórico de la consolidación y deformación alrededor de columnas de grava. Tesis Doctoral. Santander: Universidad de Cantabria.

(36) Castro, J. y Sagaseta, C. (2011). Consolidation and deformation around stone columns: Numerical evaluation of analytical solutions. Computers and Geotechnics, 38(3): 354-362. https://doi.org/10.1016/j.compgeo.2010.12.006 